\title{
Vibrational Spectra of Dense Molecular Fluids in a Laser-Heated DAC: Implications to Shock Compressed Fluids
}

\author{
B. J. Baer, C.-S. Yoo
}

This article was submitted to Shock Compression of Condensed Matter, Portland, Oregon, 07/20/2003 - 07/25/2003

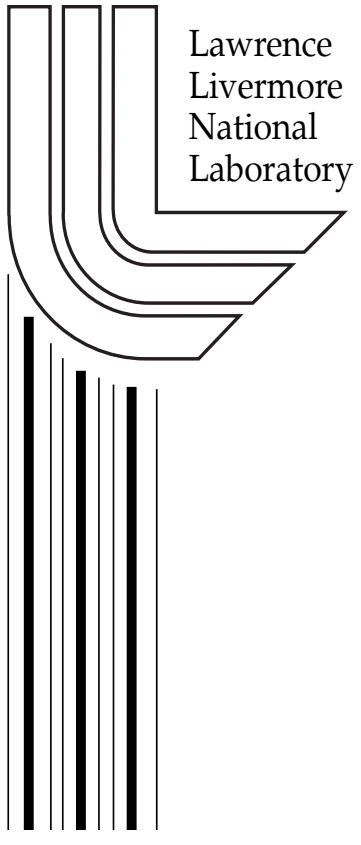

July 16, 2003 
This document was prepared as an account of work sponsored by an agency of the United States Government. Neither the United States Government nor the University of California nor any of their employees, makes any warranty, express or implied, or assumes any legal liability or responsibility for the accuracy, completeness, or usefulness of any information, apparatus, product, or process disclosed, or represents that its use would not infringe privately owned rights. Reference herein to any specific commercial product, process, or service by trade name, trademark, manufacturer, or otherwise, does not necessarily constitute or imply its endorsement, recommendation, or favoring by the United States Government or the University of California. The views and opinions of authors expressed herein do not necessarily state or reflect those of the United States Government or the University of California, and shall not be used for advertising or product endorsement purposes. 


\title{
VIBRATIONAL SPECTRA OF DENSE MOLECULAR FLUIDS IN A LASER-HEATED DAC: IMPLICATIONS TO SHOCK COMPRESSED FLUIDS
}

\author{
Bruce J. Baer and Choong-Shik Yoo \\ H-Division, Lawrence Livermore National Laboratory, University of California, Livermore, CA 94566
}

\begin{abstract}
Recent technical advances have made it possible to obtain very useful spectroscopic information about simple molecules at temperatures and pressures exceeding $2000 \mathrm{~K}$ and $10 \mathrm{GPa}$ inside a diamond-anvil cell, which is well above any melting point for such systems. This is accomplished by obtaining vibrational spectra via Coherent Anti-Stokes Raman Spectroscopy in conjunction with CW laser heating using a tungsten toroid as a laser target. By the simultaneous use of these techniques, vibrational spectra with relatively high signal to noise can be obtained despite the enormous thermal background generated by the incandescence of extremely hot laser heated material. Temperatures can be measured not only by fitting the Planck radiation to a graybody, but by the spectroscopic evidence of a Boltzmann distribution of molecules in their vibrationally excited quantum levels. Additionally, this technique allows for obtaining data at pressures and temperatures outside the region between the shock hugoniot and isentrope, complementing shock wave experiments.
\end{abstract}

\section{INTRODUCTION}

The structure, thermodynamics, energy transfer and chemical reactivity of high density fluids is important for the study of explosive detonations, planetary interiors and use in novel material syntheses. There are few accounts giving detailed spectroscopic information of materials under extreme conditions in the scientific literature. Such information is necessary to adequately understand and model the molecular potentials of even simple molecules when in a vibrationally excited state at high density. Furthermore, the question of molecular dissociation can be addressed by observing the changes in the potential with increasing pressure.

The vibrational spectra of simple molecules such as $\mathrm{N} 2, \mathrm{O} 2$, or $\mathrm{CO}$ at high temperatures $(>2000 \mathrm{~K})$ and pressures (>10 GPa) have previously been obtained under shock conditions for both the pure systems [1-3] and their mixtures. [4-7] However, in total, these studies are few and come from a single source. The difficulty of obtaining spectra under shock conditions is due to the brevity of time under which the sample is compressed and its somewhat restricted geometry. For the diamond-anvil cell, unlike the shock experiments, achieving equilibrium high temperatures is the greatest obstacle. In each experiment though, a technique to overcome the large thermal background is vital to obtaining satisfactory signal to noise.

Our experiments have, so far, focussed on nitrogen. The highest temperature previously reported for which vibrational spectra of nitrogen in a diamond-anvil cell has been observed is about 900K. [8] While this technical achievement was difficult to accomplish, much higher temperatures are needed if molecular potentials are to be determined. Also, this is in dramatic comparison to the highest temperature for vibrational spectra of nitrogen in a shock experiment - which is 4400K. [1] 


\section{EXPERIMENTAL PROCEDURE}

For diamond-anvil cell (DAC) samples, high temperatures $(>2000 \mathrm{~K})$ are achieved using laser heating. Since most simple molecules are not likely to electronically or vibrationally couple to available laser light, given that many have no dipole moment or their electronic transitions are in the ultraviolet, direct heating of a sample with a powerful CW laser is unlikely. Therefore, an indirect heating method must be used. Also, it is critical that uniform temperatures are achieved within a sample. While this occurs automatically behind the shock front of a shock experiment, a laser heated sample in a diamond-anvil cell often has an extremely high temperature gradient since the diamonds, the best known thermal conductor, are near ambient temperature.

Typically, a DAC sample will be placed inside the aperture of a metal gasket and compressed between the culets of two opposing modified brilliant cut anvils. Although the dimensions of the aperture can vary somewhat, it is typically 100 microns in diameter and 50 microns in depth at about $10 \mathrm{GPa}$. Extremely hard metals such as rhenium are used for the gasket as it will support the thickest samples. For measuring the pressure, the wavelength of the luminescence of highly calibrated materials is used. Very small ruby chips $(<10 \mu \mathrm{m})$ are usually placed inside the gasket aperture for this purpose. However, it is better to use $\mathrm{Sm}^{2+}: \mathrm{SrB}_{4} \mathrm{O}_{7}$ chips [9] instead, since there is virtually no wavelength dependence on the emission with temperature.

For a sample to be laser heated, we need to insert a target for the laser to heat. The target should be chemically inert, able to withstand extremely high temperatures, and absorb the laser light we wish to use. We have fabricated toroids made of tungsten using electric discharge machining for the target. The toroids typically have a $90 \mu \mathrm{m}$ outer diameter, a $25 \mu \mathrm{m}$ inner diameter, and are $15 \mu \mathrm{m}$ thick. The toroid is placed in the gasket aperture so that it does not touch the diamond surface where the heating laser beam will enter. It is best to have at least 10 microns spacing the two. A Nd:YLF laser capable of a maximum power of $55 \mathrm{~W}$ in $\mathrm{TEM}_{01^{*}}$ mode is focussed over the front surface area of the toroid. It is important not to focus the laser too tightly since even tungsten can melt given a sufficiently power density. Our experience with nitrogen samples is that a very thin tungsten nitride film slowly forms coats the toroid when it is sufficiently heated. Although this process makes the toroid brittle, it renders it relatively inert and helps make the heating more uniform since the reflectivity of the toroid is markedly reduced. The heated toroid is still useful as a graybody for temperature measurements since the coating is relatively thin. As long as the toroid aperture is sufficiently small, the volume within it should be at roughly uniform temperatures. It is this miniscule volume $(<10$ picoliters) that must be probed spectroscopically. It is vital that the toroid aperture is kept clear of anything but the sample.

To acquire vibrational spectra of nitrogen, Coherent Anti-Stokes Raman Spectroscopy (CARS) is employed. This is performed while the sample is heated with the Nd:YLF laser. (i.e.:insitu) This technique has a number of advantages over classical Raman Spectoscopy. The most important advantage is the ability to spatially, and even temporally, discriminate the signal from the Planck background. CARS is a four wave mixing process in which two lasers set at two different colors are used.

For our broadband CARS experiments, we use a mode locked Q-switch Nd:YAG laser. This laser produces a train of about 60 pulses, each pulse is $\sim 180 \mathrm{ps}$ long and each is sequentially separated by $\sim 12 \mathrm{~ns}$ at a repetition rate of $1 \mathrm{KHz}$. The laser wavelength is $1064 \mathrm{~nm}$. All of the pulses in the train are frequency doubled to $532 \mathrm{~nm}$. One pulse is selected as the narrow band pump laser $\left(\omega_{p}\right)$ and the rest are use to synchronously pump a dye laser using Rhodamine 640 in methanol which lases broadband $\left(\sim 200 \mathrm{~cm}^{-1}\right)$ near $607 \mathrm{~nm}\left(\omega_{\mathrm{s}}\right.$. $)$ The dye laser is intracavity dumped so that a single pulse about 30 ps long can be sent to the sample at the same time the $532 \mathrm{~nm}$ pulse $\left(\omega_{\mathrm{p}}\right)$ arrives.

When a photon from each laser crosses within the sample and they have a difference in energy resonant with a nitrogen vibration the interaction can set up a coherently excited vibrational state. This state has a very short dephasing time even though the vibrational lifetime is very long. Within the coherence lifetime another photon $\left(\omega_{\mathrm{p}}\right)$ can stimulate the CARS process resulting in a photon with a frequency $\omega_{\mathrm{as}}=2 \omega_{\mathrm{p}}-\omega_{\mathrm{s}}$. Additionally, there is a non-resonant contribution to the CARS process which makes the spectral peaks asymmetrical. The 
pulses typically have megawatt peak powers and are focussed down to a spot size near to that of the toroid aperture. Because the experiment is done coherently and the sample is much larger than the wavelength of light used, the momentum of the photons is conserved and the anti-stokes pulse comes out in a known direction and can be spatially filtered. The Planck radiation, by contrast, comes out at all directions.

\section{RESULTS AND DISCUSSION}

Figure 1 represents typical data for a sample of nitrogen at $2000 \mathrm{~K}$ which was heated from an initial pressure of $10.3 \mathrm{GPa}$ at ambient temperature.

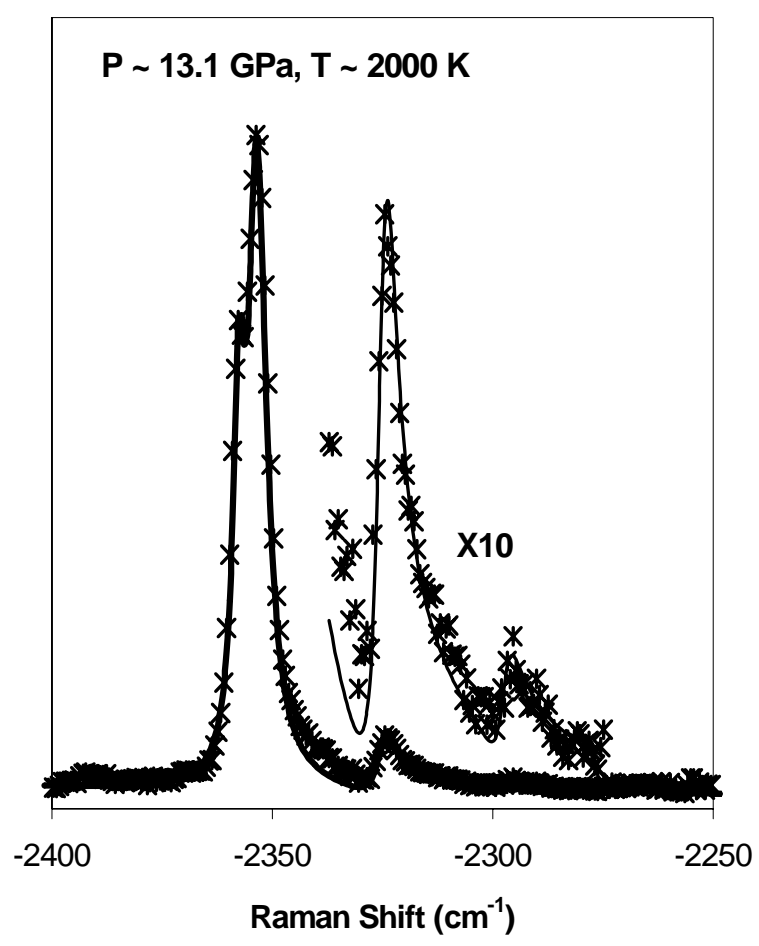

Figure 1: CARS spectrum of nitrogen. The data is represented by asterisks and the data fit is the solid line.

The data in the figure has been corrected by dividing out the frequency dependence of the dye laser $\left(\omega_{\mathrm{s}}\right)$ intensity from the raw data intensity. (i.e.: what the spectrum would look like if the dye laser output were spectrally flat.) The shoulder to the left of the ground state peak is from relatively unheated nitrogen in front of and behind the toroid. Fortunately, the hot bands have very little contribution from the cooler nitrogen. The first and second excited states can be clearly seen, confirming that this sample is indeed hot within the toroid aperture. The data has been fit using the equation below. The subscript $\mathrm{j}$ is the vibrational level and $\omega_{\mathrm{p}}$ and $\omega_{\mathrm{s}}$ are the pump and stokes frequencies of the laser, respectively. The susceptibilities are represented as $\chi$ with the pk and $\mathrm{nr}$ superscripts being the third order peak and nonresonant susceptibility, respectively. $\Gamma$ is the HWHM linewidth. This equation has been simplified from reference 1 .

$$
I_{a s} \propto I_{s} \times\left[\begin{array}{l}
\left(\sum_{j} \frac{\Gamma_{j} \chi_{j}^{p k}\left(\omega_{j}-\omega_{p}+\omega_{s}\right)}{\left(\omega_{j}-\omega_{p}+\omega_{s}\right)^{2}+\Gamma_{j}^{2}}+\chi^{n r}\right)^{2} \\
+\left(\sum_{j} \frac{\Gamma_{j}^{2} \chi_{j}^{p k}}{\left(\omega_{j}-\omega_{p}+\omega_{s}\right)^{2}+\Gamma_{j}^{2}}\right)^{2}
\end{array}\right]
$$

The fitting results in an error in pressure of approximately $0.7 \mathrm{GPa}$ and an error in temperature of about $200 \mathrm{~K}$. Which are the same errors as one would get using the luminescence and graybody methods that are standard for DAC research.

For this fit, the energies of $\omega_{\mathrm{j}}$ decreased by $\sim 28 \mathrm{~cm}^{-1}$ for the first excited level and $\sim 27.5 \mathrm{~cm}^{-1}$ for the second. This is very close to the results obtained in reference 1 . If the potential becomes shallower (i.e.: the triple bond is breaking) due to increasing pressure, as suggested by shock experiments, [10] it can be directly observed by this method. Experiments at higher pressures are currently being run in order to determine if this can be observed in the DAC.

\section{CONCLUSIONS}

This work demonstrates that there are methods to 'fill in' the gap between static and high pressure experiments. The ability to acquire vibrational spectra under conditions usually associated with shock experiments has been clearly demonstrated on nitrogen samples. Using a combination of laser heating tungsten toroid targets and coherent anti- 
Stokes spectroscopy we can obtain vibrational spectra of dense fluids at high temperature. Currently, improvements to this technique resulting in higher temperatures and pressures are underway. As yet, temperatures of $\sim 2000 \mathrm{~K}$ and pressure of $\sim 15 \mathrm{GPa}$ have been achieved. Furthermore, it should be possible to achieve conditions outside the range of shock conditions. (i.e.: between the hugoniot and isentrope.)

\section{ACKNOWLEDGMENT}

We greatly appreciate the efforts of Ken Visbeck for his valued technical assistance and the advice of $\mathrm{R}$. Boehler concerning the toroid fabrication technique. This work has been supported by the LDRD (00-ERD-024) and PDRP programs. This work was performed under the auspices of the U.S. Department of Energy by the University of California, Lawrence Livermore National Laboratory under contract No. W-7405-Eng-48.

\section{REFERENCES}

1. Moore, D. S., Schmidt, S. C., Shaw, M. S., and Johnson, J. D., J. Chem. Phys. 90, 1368-1376 (1989).

2. Schmidt, S. C., Moore, D. S., Shaw, M. S., and Johnson, J. D., J. Chem. Phys. 91, 6765-6771 (1989).

3. Moore, D. S., Schmidt, S. C., and Shaw, M. S., and Johnson, J. D., J. Chem. Phys. 95, 56035608 (1991).

4. Schmidt, S. C., and Moore, D. S., Acc. Chem. Res. 25, 427-432 (1992).

5. Schmidt, S. C., Moore, D. S., and Shaw, M. S., J. Chem. Phys. 107, 325-336 (1997).

6. Schmidt, S. C., Moore, D. S., Shaw, M. S., and Johnson, J. D., J. Chem. Phys. 98, 9379-9388 (1993).

7. Schmidt, S. C., Moore, D. S., and Shaw, M. S., J. Chem. Phys. 101, 3488-3494 (1994).

8. Zinn, A. S., Schiferl, D., and Nicol, M. F., J. Chem. Phys. 87, 1267-1271 (1987).

9. Datchi, F., LeToullec, R., and Loubeyre, P., J. Appl. Phys. 81, 3333-3339 (1997).
10. Nellis, W. J., Holmes, N. C., Mitchell, A. C., and van Thiel, M., Phys. Rev. Lett. 53, 16611664 (1984). 